\title{
BROWNIAN COAGULATION
}

\author{
J.R. NORRIS *
}

\begin{abstract}
We consider a stochastic particle model for coagulating particles, whose free motion is Brownian, with diffusivity given by Einstein's law. We present in outline a derivation from this model of a spatially inhomogeneous version of Smoluchowski's coagulation equation. Some analytic results on existence, uniqueness and mass conservation for the limit equation are also presented.
\end{abstract}

\section{Introduction}

We will consider two models for the evolution of a cloud of particles, of various sizes, subject to diffusion and to coagulation on collision. The first model is a stochastic system of spherical particles in $\mathbb{R}^{d}, d \geqslant 3$, starting with $N$ particles, centered at positions $x_{1}, \ldots, x_{N}$ in $\mathbb{R}^{d}$ and having masses $y_{1} / N, \ldots, y_{N} / N \in(0, \infty)$. After $k$ collisions we will have $N-k$ particles, each characterized by a position $x$, mass $y / N$, radius $r / N^{1 /(d-2)}$ and (isotropic) diffusivity $a$. We assume that $y$ determines $r$ and $a$ through $y=r^{d}$ and $a=r^{d-1} / y$. The first of these relations is a homogeneity assumption. The second is Einstein's law for the diffusivity of physical Brownian motion. Each particle performs an independent Brownian motion according to its own diffusivity. When two particles collide, we replace them by a single particle having the same total mass and centre of mass. These rules determine a process of normalized empirical measures $\left(\mu_{t}^{N}\right)_{t \geqslant 0}$ on $\mathbb{R}^{d} \times(0, \infty)$, starting from

$$
\mu_{0}^{N}=\frac{1}{N} \sum_{i=1}^{N} \delta_{\left(x_{i}, y_{i}\right)}
$$

which we call Brownian coagulation.

The second model is a deterministic process of measures $\left(\mu_{t}\right)_{t \geqslant 0}$ on $\mathbb{R}^{d} \times(0, \infty)$, satisfying the following evolution equation

$$
\dot{\mu}_{t}=\frac{1}{2} a \Delta \mu_{t}+K\left(\mu_{t}\right) .
$$

Here we assume that each measure is given by a density $\mu_{t}(d x, d y)=\mu_{t}(x, d y) d x$ on $\mathbb{R}^{d}$; the diffusivity $a=a(y)=y^{-1 / d}$ as above and $\Delta=\Delta_{x}$. The coagulation term $K\left(\mu_{t}\right)$ is given at each $\mu=\mu_{t}(x, \cdot)$ by

$$
\langle f, K(\mu)\rangle=\frac{1}{2} \int_{(0, \infty)^{2}}\left\{f\left(y+y^{\prime}\right)-f(y)-f\left(y^{\prime}\right)\right\} K\left(y, y^{\prime}\right) \mu(d y) \mu\left(d y^{\prime}\right)
$$

for suitable test-functions $f$, where

$$
K\left(y, y^{\prime}\right)=c_{d}\left(a+a^{\prime}\right)\left(r+r^{\prime}\right)^{d-2}
$$

$c_{d}$ is the capacity of the unit ball and $r=r(y)=y^{1 / d}$ as above.

The spatially homogeneous, discrete mass case of this equation was derived, for $d=3$, by Smoluchowski [11], starting from an account of particle motion corresponding to the stochastic model described above. We will describe two different approaches

*Statistical Laboratory, Centre for Mathematical Sciences, Wilberforce Road, Cambridge, CB3 0WB, UK (j.r.norris@statslab.cam.ac.uk). 
to Smoluchowski's equation. The first allows us to construct, for suitable initial data, a small-time solution $\left(\mu_{t}\right)_{t<t_{0}}$ and to prove that $\mu_{t}^{N} \rightarrow \mu_{t}$ weakly in probability on $\mathbb{R}^{d} \times(0, \infty)$ for all $t<t_{0}$. We could describe the behaviour of mass under coagulation in our model as $\left(y, y^{\prime}\right) \rightarrow y+y^{\prime}$ : in previous work Lang and Nguyen [3] treated the case $(1,1) \rightarrow 1$ and Sznitman [9], [10] the case $(1,1) \rightarrow 0$. The sort of limit considered is similar to Lanford's derivation [2] of the Boltzmann equation.

The second approach draws on a truncation argument developed in [8],[7]. We establish long-time existence and uniqueness for the coagulation-diffusion equation, along with the natural property of mass conservation

$$
\int_{\mathbb{R}^{d} \times(0, \infty)} y \mu_{t}(x, d y) d x=\int_{\mathbb{R}^{d} \times(0, \infty)} y \mu_{0}(x, d y) d x, \quad t \geqslant 0 .
$$

For related recent work see Amann [1] and Laurençot and Mischler [4], [5]. Full details of the results presented in this paper will appear elsewhere.

\section{Weak convergence in small time}

The basic idea is to consider a process in which new particles are created on collision but the old ones are not deleted. We disregard any collision between particles having one or more initial particles in common, also any collision which is not the first between the colliding particles. Write $\left(\nu_{t}^{N}\right)_{t \geqslant 0}$ for the normalized empirical measure on $\mathbb{R}^{d} \times(0, \infty)$. The corresponding evolution equation is

$$
\dot{\nu}_{t}=\frac{1}{2} a \Delta \nu_{t}+K^{+}\left(\nu_{t}\right)
$$

where

$$
\left\langle f, K^{+}(\nu)\right\rangle=\frac{1}{2} \int_{(0, \infty)^{2}} f\left(y+y^{\prime}\right) K\left(y, y^{\prime}\right) \nu(d y) \nu\left(d y^{\prime}\right) .
$$

We interpret this equation in the weak sense

$$
\nu_{t}=P_{t} \nu_{0}+\int_{0}^{t} P_{t-s} K^{+}\left(\nu_{s}\right) d s
$$

where

$$
P_{t} \nu(x, d y)=\int_{\mathbb{R}^{d}} p\left(a(y) t, x, x^{\prime}\right) \nu\left(x^{\prime}, d y\right) d x^{\prime}
$$

and $p\left(t, x, x^{\prime}\right)=(2 \pi t)^{-d / 2} e^{-\left|x-x^{\prime}\right|^{2} / 2 t}$. This weak equation has a minimal nonnegative solution $\left(\nu_{t}\right)_{t \geqslant 0}$ for any given initial kernel $\nu_{0}(x, d y)$, but this may rapidly, or even instantaneously, become infinite. However, for suitable initial conditions we have good small-time behaviour. We assume that $\mu_{0}(x, d y)=\nu_{0}(x, d y)=\phi(x) \delta_{1}(d y)$ for all $x$, where $\phi$ is a probability density function with $\phi \leqslant 1$. We assume also that our initial particles are obtained as a random sample of size $N$ from $\mu_{0}$.

Proposition 2.1. There is a constant $A<\infty$ such that, for all $t \geqslant 0, n \in \mathbb{N}$ and all $x$ we have $\nu_{t}(x,\{n\}) \leqslant(A t)^{n-1}$.

We can show that $\nu_{t}^{N} \rightarrow \nu_{t}$ weakly in probability on $\mathbb{R}^{d} \times\{n\}$ for all $n \in \mathbb{N}$ as $N \rightarrow \infty$ using the following result for Brownian motion. See, for example, Le Gall [6] for related results. 
Proposition 2.2. For $i=1,2$, let $X^{i}$ be a Brownian motion in $\mathbb{R}^{d}$ of diffusivity $a_{i}$ starting from $x_{i}$, where $x_{1} \neq x_{2}$. Set

$$
T=\inf \left\{t \geqslant 0:\left|X_{t}^{1}-X_{t}^{2}\right|=\left(r_{1}+r_{2}\right) / N^{1 /(d-2)}\right\}
$$

and let $\left(X_{t}\right)_{t \geqslant T}$ be a Brownian motion of diffusivity a starting from $\left(y_{1} X_{T}^{1}+y_{2} X_{T}^{2}\right) /\left(y_{1}\right.$ $\left.+y_{2}\right)$ at time $T$. Then for any bounded measurable function $f$ on $\mathbb{R}^{d}$

$$
N \mathbb{E}\left(f\left(X_{t}\right) 1_{T \leqslant t}\right) \rightarrow K \int_{0}^{t} \int_{\mathbb{R}^{d} \times \mathbb{R}^{d}} p\left(a_{1} s, x_{1}, z\right) p\left(a_{2} s, x_{2}, z\right) p(a(t-s), z, x) f(x) d z d x d s .
$$

where $K=c_{d}\left(a_{1}+a_{2}\right)\left(r_{1}+r_{2}\right)^{d-2}$.

This in itself is not sufficient to prove convergence of our original particle system. For this we must establish convergence of the process $\left(\nu_{t}^{N}\right)_{t \geqslant 0}$ at a sufficiently detailed level that we can identify which particles should have been deleted, even in the limit. This requires that we retain in the current state of each particle the historical tree of all positions and masses of all constituent initial particles, all particles which have collided with constituent particles, all particles which have collided with the colliding particles, and so on.

We refer to the appendix for an explanation of the notation we use for trees. For $i=1, \ldots, N$, set $S_{i}=0$ and let $\left(X_{t}^{i}\right)_{t \geqslant 0}$ be a Brownian motion in $\mathbb{R}^{d}$, starting from $x_{i}$ of diffusivity $a\left(y_{i}\right)$. For a tree $i=\left\{i_{1}, i_{2}\right\} \in I=\mathbb{T}(\{1, \ldots, N\})$, set

$$
S_{i}=\inf \left\{t \geqslant S_{i_{1}} \vee S_{i_{2}}:\left|X_{t}^{i_{1}}-X_{t}^{i_{2}}\right|=\left(r\left(y_{i_{1}}\right)+r\left(y_{i_{2}}\right)\right) / N^{1 /(d-2)}\right\}
$$

and let $\left(X_{t}^{i}\right)_{t \geqslant S_{i}}$ be a Brownian motion in $\mathbb{R}^{d}$ starting from $\left(y_{i_{1}} X_{S_{i}}^{i_{1}}+y_{i_{2}} X_{S_{i}}^{i_{2}}\right) /\left(y_{i_{1}}+y_{i_{2}}\right)$ at time $S_{i}$ of diffusivity $a\left(y_{i}\right)$, where $y_{i}=y_{i_{1}}+y_{i_{2}}$. For $i=\left\{i_{1}, i_{2}\right\}$ and $j=\left(i_{1}, i_{2}\right)$ in $I^{(2)}=\mathbb{T}^{(2)}(\{1, \ldots, N\})$, set $S_{i}=S_{j}=S_{\pi(i)}$ if $S_{\pi(i)} \geqslant S_{i_{1}} \vee S_{i_{2}}$ and $S_{i}=S_{j}=\infty$ otherwise. For $i=1, \ldots, N$ and $t \geqslant 0$, set $\xi_{t}^{i}=\left(\left.X^{i}\right|_{[0, t]}, y_{i}\right)$ and, recursively for $i=\left\{i_{1}, i_{2}\right\}$ and $j=\left(i_{1}, i_{2}\right)$ in $I^{(2)}$, for $t \geqslant s=S_{i}$, set $\xi_{t}^{i}=\left(s,\left\{\xi_{s}^{i_{1}}, \xi_{s}^{i_{2}}\right\},\left.X^{\pi(i)}\right|_{[s, t]}\right)$ and $\xi_{t}^{j}=\left(s,\left(\xi_{s}^{i_{1}}, \xi_{s}^{i_{2}}\right),\left.X^{\pi(j)}\right|_{[s, t]}\right)$. Define a measure $\tilde{\nu}_{t}^{N}$ on $A^{(2)}[0, t]$ by

$$
\tilde{\nu}_{t}^{N}=\frac{1}{N} \sum_{i \in I^{(2)}} \delta_{\xi_{t}^{i}} 1_{S_{i} \leqslant t}
$$

Then we have

$$
\nu_{t}^{N}=\left(\tilde{\nu}_{t}^{N} 1_{A[0, t]}\right) \circ\left(x_{t}, y_{t}\right)^{-1}
$$

where $\left(x_{t}, y_{t}\right): A^{(2)}[0, t] \rightarrow \mathbb{R}^{d} \times(0, \infty)$ is the endpoint map.

We now proceed to define the corresponding limit measure on the set of path-trees $A^{(2)}[0, t]$. Define for $\xi=(\omega, y) \in A_{1}[0, t]$

$$
\tilde{\nu}_{t}(x, d \xi)=\int_{x_{0} \in \mathbb{R}^{d}} \mu_{0}\left(x_{0}, d y\right) d x_{0} \beta_{y}^{0, x_{0} ; t, x}(d \omega)
$$

where $\beta_{y}^{0, x_{0} ; t, x}$ is the un-normalized Brownian bridge on $C\left([0, t], \mathbb{R}^{d}\right)$, starting from $x_{0}$ and ending at $x$, with diffusivity $a(y)$. Then, recursively for $\xi=\left(s,\left\{\xi_{1}, \xi_{2}\right\}, \omega\right)$, set

$$
\tilde{\nu}_{t}(x, d \xi)=\frac{1}{2} \int_{z \in \mathbb{R}^{d}} \tilde{\nu}_{s}\left(z, d \xi_{1}\right) \tilde{\nu}_{s}\left(z, d \xi_{2}\right) K\left(y_{s}\left(\xi_{1}\right), y_{s}\left(\xi_{2}\right)\right) d s \beta_{y_{t}(\xi)}^{s, z ; t, x}(d \omega) d z .
$$


The formula for $\xi=\left(s,\left(\xi_{1}, \xi_{2}\right), \omega\right)$ is the same except that the factor $1 / 2$ is absent. Then

$$
\nu_{t}(x, d y)=\left(\tilde{\nu}_{t}(x, \cdot) 1_{A[0, t]}\right) \circ y_{t}^{-1}(d y)
$$

Proposition 2.3. We have $\tilde{\nu}_{t}^{N} \rightarrow \tilde{\nu}_{t}$ weakly in probability on $A_{\tau}[0, t]$ for all $\tau \in \mathbb{T}^{(2)}$ as $N \rightarrow \infty$.

Fix $t<t_{0}=A^{-1}$ and define measures $\rho_{t}^{N}$ and $\rho_{t}$ on $A^{(2)}[0, t]$ by

$$
\rho_{t}^{N}=\frac{1}{N} \sum_{i \in I^{(2)}} \delta_{\xi_{t}^{i}} 1_{\left\{S_{i} \leqslant t \text { and } S_{j}>t \text { for all } j \in N(i)\right\}}
$$

and

$$
\rho_{t}(x, d \xi)=\exp \left(-\int_{\Delta(\xi)} \lambda_{s}\left(x_{s}(\xi), y_{s}(\xi)\right) d s\right) \nu_{t}(x, d \xi)
$$

where

$$
\lambda_{t}(x, y)=\int_{(0, \infty)} K\left(y, y^{\prime}\right) \nu_{t}\left(x, d y^{\prime}\right)
$$

Then

$$
\rho_{t}(x, d \xi)=\int_{A[0, t]} \nu_{t}\left(x, d \xi_{0}\right) \beta_{t}\left(\xi_{0}, d \xi\right)
$$

where $\beta_{t}\left(\xi_{0}, d \xi\right)$ is the law of the total tree of a typed branching process starting from $\xi_{0}$, where the offspring of $\xi_{0}$ form a Poisson random measure on $A\left(\xi_{0}\right)$ having intensity measure $K\left(y_{s}\left(\xi_{0}\right), y_{s}\left(\xi^{\prime}\right)\right) \nu_{s}\left(x_{s}\left(\xi_{0}\right), d \xi^{\prime}\right) d s$. This branching process is subcritical as a consequence of Proposition 2.1.

Proposition 2.4. We have $\rho_{t}^{N} \rightarrow \rho_{t}$ weakly in probability on $A^{(2)}[0, t]$ as $N \rightarrow \infty$.

The proof begins by writing

$$
1_{\left\{S_{i} \leqslant t \text { and } S_{j}>t \text { for all } j \in N(i)\right\}}=\sum_{n=0}^{\infty}(-1)^{n} \sum_{j \in N_{n}(i)} 1_{S_{j} \leqslant t}
$$

and exploits the fact that the partial sums of the series on the right successively overestimate and underestimate the limit.

Now define measures $\tilde{\mu}_{t}^{N}$ and $\tilde{\mu}_{t}$ on $A[0, t]$ by

$$
\tilde{\mu}_{t}^{N}=\left(\rho_{t}^{N} 1_{\tau \in \mathbb{S}}\right) \circ \pi^{-1}, \quad \tilde{\mu}_{t}=\left(\rho_{t} 1_{\tau \in \mathbb{S}}\right) \circ \pi^{-1} .
$$

The atoms of $\rho_{t}^{N}$ comprise all the maximal trees of particle histories which could result in a particle at time $t$. The particle is actually formed if and only if its maximal tree $i$ is a surviving tree (see the appendix). When $\tau(i) \notin \mathbb{S}$ some unwanted collision prevents the $i$-particle from forming. Hence we obtain

$$
\mu_{t}^{N}=\tilde{\mu}_{t}^{N} \circ\left(x_{t}, y_{t}\right)^{-1}
$$

Theorem 2.5. We have $\tilde{\mu}_{t}^{N} \rightarrow \tilde{\mu}_{t}$ weakly in probability on $A[0, t]$ as $N \rightarrow \infty$. 
We now show by a branching process argument that $\tilde{\mu}_{t}$ satisfies an equation which implies that $\mu_{t}$ solves Smoluchowski's equation. For $\xi_{0} \in A[0, t]$, write $p\left(\xi_{0}\right)$ for the probability that its total tree $\xi$ survives, that is $\tau(\xi) \in \mathbb{S}$. Then for $\xi_{0}=\left(s,\left\{\xi_{0}^{1}, \xi_{0}^{2}\right\}, \omega\right)$ with $y_{t}\left(\xi_{0}\right)=y$,

$$
p\left(\xi_{0}\right)=p\left(\xi_{0}^{1}\right) p\left(\xi_{0}^{2}\right) \exp \left(-\int_{s}^{t} \int_{A[0, r]} K\left(y, y_{r}\left(\xi^{\prime}\right)\right) p\left(\xi^{\prime}\right) \tilde{\nu}_{r}\left(\omega_{r}, d \xi^{\prime}\right) d r\right) .
$$

Hence

$$
\begin{aligned}
\tilde{\mu}_{t}\left(x, d \xi_{0}\right)= & p\left(\xi_{0}\right) \tilde{\nu}_{t}\left(x, d \xi_{0}\right) \\
= & \int_{R^{d}} p\left(\xi_{0}^{1}\right) \tilde{\nu}_{t}\left(z, d \xi_{0}^{1}\right) p\left(\xi_{0}^{2}\right) \tilde{\nu}_{t}\left(z, d \xi_{0}^{2}\right) K\left(y_{s}\left(\xi_{0}^{1}\right), y_{s}\left(\xi_{0}^{2}\right)\right) d s \\
& \quad \times \exp \left(-\int_{s}^{t} \int_{A[0, r]} K\left(y, y_{r}\left(\xi^{\prime}\right)\right) p\left(\xi^{\prime}\right) \tilde{\nu}_{r}\left(\omega_{r}, d \xi^{\prime}\right) d r\right) \beta_{y}^{s, z ; t, x}(d \omega) d z \\
= & \int_{R^{d}} \tilde{\mu}_{t}\left(z, d \xi_{0}^{1}\right) \tilde{\mu}_{t}\left(z, d \xi_{0}^{2}\right) K\left(y_{s}\left(\xi_{0}^{1}\right), y_{s}\left(\xi_{0}^{2}\right)\right) d s \\
& \quad \times \exp \left(-\int_{s}^{t} \int_{A[0, r]} K\left(y, y_{r}\left(\xi^{\prime}\right)\right) \tilde{\mu}_{r}\left(\omega_{r}, d \xi^{\prime}\right) d r\right) \beta_{y}^{s, z ; t, x}(d \omega) d z .
\end{aligned}
$$

\section{Long-time existence, uniqueness and mass conservation}

We will take $d=3$, and set $w(y)=y^{1 / 3}+y^{-1 / 3}$. Note that

$$
K\left(y, y^{\prime}\right)=\left(y^{1 / 3}+y^{\prime / 3}\right)\left(y^{-1 / 3}+y^{\prime-1 / 3}\right) \leqslant w(y) w\left(y^{\prime}\right)
$$

and

$$
w\left(y+y^{\prime}\right) p\left(a\left(y+y^{\prime}\right) t, x, x^{\prime}\right) \leqslant w(y) p\left(a(y) t, x, x^{\prime}\right)+w\left(y^{\prime}\right) p\left(a\left(y^{\prime}\right) t, x, x^{\prime}\right) .
$$

Suppose we have a particle of mass $y$ at position $x$ at time $t$ and two particles of masses $z, z^{\prime}$ at $x^{\prime}$ at time 0 . Then

$$
w(y)\left\{w(z) p\left(a(z) t, x, x^{\prime}\right)+w\left(z^{\prime}\right) p\left(a\left(z^{\prime}\right) t, x, x^{\prime}\right)\right\}
$$

is an upper bound for the rate at which the first particle coagulates with one of the others which remains valid if the $z, z^{\prime}$ particles happen to coagulate. This (imprecisely formulated) observation is the key to the method by which we treat Smoluchowski's equation. It allows a truncation argument where we do not keep track of coagulations between large particles, safe in the knowledge that we can bound, regardless of these coagulations, the rate at which small particles are captured by large ones. Assume that

$$
\int_{\mathbb{R}^{3} \times(0, \infty)}\left(y^{4 / 3}+y^{-1 / 3}\right) \mu_{0}(x, d y) d x<\infty
$$

and that $\mu_{0}(x, d y) \leqslant \mu_{0}^{*}(d y)$ for all $x \in \mathbb{R}^{3}$, with

$$
\int_{(0, \infty)}\left(y^{2 / 3}+y^{-2 / 3}\right) \mu_{0}^{*}(d y)<\infty
$$


THEOREM 3.1. There exists within the class of kernels $\left(\mu_{t}\right)_{t \geqslant 0}$ such that

$$
\int_{0}^{t} \int_{(0, R]} y^{1 / 2} P_{t-s} K^{+}\left(\mu_{s}\right)(x, d y) d s<\infty
$$

for all $t \geqslant 0, x \in \mathbb{R}^{3}$ and $R<\infty$, a unique kernel $\left(\mu_{t}\right)_{t \geqslant 0}$ satisfying

$$
\mu_{t}+\int_{0}^{t} P_{t-s} K^{-}\left(\mu_{s}\right) d s=P_{t} \mu_{0}+\int_{0}^{t} P_{t-s} K^{+}\left(\mu_{s}\right) d s, \quad t \geqslant 0 .
$$

Moreover

(i)

$$
\int_{\mathbb{R}^{3} \times(0, \infty)} y \mu_{t}(x, d y) d x=\int_{\mathbb{R}^{3} \times(0, \infty)} y \mu_{0}(x, d y) d x,
$$

(ii)

$$
\dot{\mu}_{t}=\frac{1}{2} a(y) \Delta \mu_{t}+K\left(\mu_{t}\right)
$$

(iii) If $\mu_{0}(x, \cdot)$ is absolutely continuous for all $x$, then so is $\mu_{t}(x, \cdot)$ for all $t \geqslant 0$.

In the remainder of this section we will explain the role of condition (3.2) and then sketch the route to existence, uniqueness and mass conservation. The inequality (3.1) implies that

$$
\left\langle w, P_{t-s} K^{+}\left(\mu_{s}\right)\right\rangle \leqslant\left\langle w, P_{t-s} K^{-}\left(\mu_{s}\right)\right\rangle
$$

for $0 \leqslant s<t$. Here $\langle.,$.$\rangle denotes the integral over (0, \infty)$ : the inequality is between functions on $\mathbb{R}^{3}$. If we knew that

$$
\int_{0}^{t}\left\langle w, P_{t-s} K^{+}\left(\mu_{s}\right)\right\rangle d s<\infty
$$

we could multiply $(3.3)$ by $w$, integrate over $(0, \infty)$ and subtract this term from both sides to obtain

$$
\left\langle w, \mu_{t}\right\rangle \leqslant\left\langle w, P_{t} \mu_{0}\right\rangle \leqslant\left\langle w, \mu_{0}^{*}\right\rangle<\infty
$$

and

$\left\|\left\langle w, \mu_{t}\right\rangle\right\|_{1} \leqslant\left\|\left\langle w, P_{t} \mu_{0}\right\rangle\right\|_{1}=\left\|\left\langle w, \mu_{0}\right\rangle\right\|_{1}<\infty$. We do not know (3.4) but by approximating $w$ suitably and using (3.2) we can arrive at the same conclusions. Thus the very weak condition (3.2) bootstraps to (3.5), which gives a useful control on the behaviour of solutions.

The existence and uniqueness of solutions is obtained by constructing a sequence of solutions to approximating equations $\left(\mu_{t}^{n}\right)_{t \geqslant 0}$ together with some auxilliary functions $\left(\eta_{t}^{n}\right)_{t \geqslant 0}$ on $\mathbb{R}^{3}$. These approximating equations involve only $K \wedge n$ and are solvable by a standard iteration scheme. They are chosen so that we have

$$
\mu_{t}^{n} \leqslant \mu_{t}^{n+1}, \quad\left\langle w, \mu_{t}^{n}\right\rangle+\eta_{t}^{n} \geqslant\left\langle w, \mu_{t}^{n+1}\right\rangle+\eta_{t}^{n+1}
$$

and indeed, for any solution $\left(\nu_{t}\right)_{t \geqslant 0}$ to $(3.2),(3.3)$ 


$$
\mu_{t}^{n} \leqslant \nu_{t}, \quad\left\langle w, \mu_{t}^{n}\right\rangle+\eta_{t}^{n} \geqslant\left\langle w, \nu_{t}\right\rangle
$$

Roughly speaking, $\mu_{t}^{n}$ corresponds to particles of mass $y \in[1 / n, n]$ that we are sure about, and $w(y)\left(\left\langle w, \mu_{t}^{n}\right\rangle+\eta_{t}^{n}\right)$ bounds the rate at which we lose these particles by coagulation, $\eta_{t}^{n}$ being due to the large particles that we have lost. The monotone limits $\mu_{t}=\lim _{n \rightarrow \infty} \mu_{t}^{n}$ and $\eta_{t}=\lim _{n \rightarrow \infty} \eta_{t}^{n}$ can be shown to satisfy

$$
\mu_{t}+\int_{0}^{t} P_{t-s}\left(K^{-}\left(\mu_{s}\right)+\eta_{s} w \mu_{s}\right) d s=P_{t} \mu_{0}+\int_{0}^{t} P_{t-s} K^{+}\left(\mu_{s}\right) d s
$$

and of course, for any solution $\left(\nu_{t}\right)_{t \geqslant 0}$,

$$
\mu_{t} \leqslant \nu_{t}, \quad\left\langle w, \mu_{t}\right\rangle+\eta_{t} \geqslant\left\langle w, \nu_{t}\right\rangle
$$

Thus existence and uniqueness both rest on showing that $\eta_{t}=0$ for all $t \geqslant 0$.

The key to this is the following a priori argument. Let $\left(\mu_{t}\right)_{t \geqslant 0}$ be a solution. Set $v(y)=y^{-1 / 3}$ and note that (3.1) holds with $w$ replaced by $w v$. Hence

$$
\left\langle w v, \mu_{t}\right\rangle \leqslant\left\langle w v, \mu_{0}^{*}\right\rangle<\infty .
$$

Note that

$$
K\left(y, y^{\prime}\right) \leqslant 2\left(w(y) v\left(y^{\prime}\right)+v(y) w\left(y^{\prime}\right)\right)
$$

and for $p(y)=p\left(a(y) t, x, x^{\prime}\right)$

$$
w^{2} p\left(y+y^{\prime}\right)-w^{2} p(y)-w^{2} p\left(y^{\prime}\right) \leqslant w(y)\left(p(y)+p\left(y^{\prime}\right)\right) w\left(y^{\prime}\right) .
$$

Act on $(3.3)$ by $P_{s}$, multiply by $w^{2}$ and integrate over $(0, \infty)$. Then, assuming all terms finite, rearrange to obtain

$$
\begin{aligned}
& \left\langle w^{2}, P_{s} \mu_{t}\right\rangle-\left\langle w^{2}, P_{s+t} \mu_{0}\right\rangle=\int_{0}^{t}\left\langle w^{2}, P_{s+t-r} K\left(\mu_{r}\right)\right\rangle d r \\
& \leqslant 2 \int_{0}^{t} \int_{\mathbb{R}^{3} \times(0, \infty)^{2}} w(y) p\left(a(y)(s+t-r), \cdot, x^{\prime}\right) w\left(y^{\prime}\right)\left(w(y) v\left(y^{\prime}\right)\right. \\
& \left.\quad+v(y) w\left(y^{\prime}\right)\right) \mu_{r}(d y) \mu_{r}\left(d y^{\prime}\right) d x^{\prime} d r \\
& \leqslant 2\left\langle w v, \mu_{0}^{*}\right\rangle \int_{0}^{t}\left(\left\|\left\langle w^{2}, P_{s+t-r} \mu_{r}\right\rangle\right\|_{\infty}+\left\|\left\langle w^{2}, \mu_{r}\right\rangle\right\|_{\infty}\right) d r
\end{aligned}
$$

so, by Gronwall's lemma,

$$
\sup _{s \geqslant 0}\left\|\left\langle w^{2}, P_{s} \mu_{t}\right\rangle\right\|_{\infty} \leqslant\left\langle w^{2}, \mu_{0}^{*}\right\rangle e^{4\left\langle w^{2}, \mu_{0}^{*}\right\rangle t} .
$$

We do not know that all terms are finite $a$ priori but a version of this argument works for $\left(\mu_{t}^{n}\right)_{t \geqslant 0}$. So we obtain (3.6) for the monotone limit $\mu_{t}=\lim _{n \rightarrow \infty} \mu_{t}^{n}$. We then get the following limiting equation for $\left(\eta_{t}\right)_{t \geqslant 0}$

$$
\eta_{t}=\int_{0}^{t}\left\langle w^{2}, P_{t-s}\left(\eta_{s} \mu_{s}\right)\right\rangle d s
$$


SO

$$
\left\|\eta_{t}\right\|_{1}=\int_{0}^{t}\left\|\left\langle w^{2}, \eta_{s} \mu_{s}\right\rangle\right\|_{1} d s \leqslant \int_{0}^{t}\left\|\left\langle w^{2}, \mu_{s}\right\rangle\right\|_{\infty}\left\|\eta_{s}\right\|_{1} d s .
$$

Since $\left\|\eta_{t}\right\|_{1}$ is non-decreasing and finite, this implies $\eta_{t}=0$ a.e. for all $t$ as required.

To establish conservativity, multiply (3.3) by $y 1_{y \leqslant R}$ and integrate over $\mathbb{R}^{3} \times(0, \infty)$ to obtain

$$
\begin{aligned}
\int_{\mathbb{R}^{3} \times(0, R]} y \mu_{0}(x, d y) d x-\int_{\mathbb{R}^{3} \times(0, R]} y \mu_{t}(x, d y) d x \\
=\int_{0}^{t} \int_{\mathbb{R}^{3} \times(0, \infty)^{2}} y 1_{y \leqslant R, y+y^{\prime}>R} K\left(y, y^{\prime}\right) \mu_{s}(x, d y) \mu_{s}\left(x, d y^{\prime}\right) d x d s .
\end{aligned}
$$

The integrand on the right converges pointwise to 0 as $R \rightarrow \infty$ and, we recall, $K\left(y, y^{\prime}\right) \leqslant w(y) w\left(y^{\prime}\right)$ and $\left\langle w, \mu_{t}\right\rangle \leqslant\left\langle w, \mu_{0}^{*}\right\rangle<\infty$. So we will obtain conservativity by dominated convergence on letting $R \rightarrow \infty$ once we can show for $u(y)=y^{2 / 3}$ that

$$
\int_{0}^{t}\left\|\left\langle u^{2}, \mu_{s}\right\rangle\right\|_{1} d s<\infty
$$

Set $u_{R}(y)=y^{2 / 3} 1_{y \leqslant R}$. Then

$$
\begin{aligned}
\left\|\left\langle u_{R}^{2}, \mu_{t}\right\rangle\right\|_{1} & =\left\|\left\langle u_{R}^{2}, P_{t} \mu_{0}\right\rangle\right\|_{1}+\int_{0}^{t}\left\|\left\langle u_{R}^{2}, P_{t-s} K\left(\mu_{s}\right)\right\rangle\right\|_{1} d s \\
& \left.\leqslant\left\|\left\langle u^{2}, \mu_{0}\right\rangle\right\|_{1}+2 \int_{0}^{t} \|\left\langle u_{R} w, \mu_{s}\right)\right\rangle\left\|_{1}\right\|\left\langle u v, \mu_{s}\right\rangle \|_{\infty} d s
\end{aligned}
$$

and $\left.\|\left\langle u_{R} w, \mu_{s}\right)\right\rangle\left\|_{1} \leqslant\right\|\left\langle u w, \mu_{0}\right\rangle \|_{1}<\infty$ and $\left\|\left\langle u v, \mu_{s}\right\rangle\right\|_{\infty} \leqslant\left\|\left\langle u v, P_{s} \mu_{0}\right\rangle\right\|_{\infty} \leqslant\left\langle u v, \mu_{0}^{*}\right\rangle$ $<\infty$. This provides the necessary estimate.

4. Appendix - trees

Let $E$ be a set. Define $\mathbb{T}_{1}(E)=E$ and for $n \geqslant 2$

$$
\mathbb{T}_{n}(E)=\left\{\left\{y_{1}, y_{2}\right\}: y_{1} \in \mathbb{T}_{k}(E), y_{2} \in \mathbb{T}_{n-k}(E), k=1, \ldots, n-1\right\}
$$

and

$$
\mathbb{T}_{n}^{(2)}(E)=\left\{\left\{y_{1}, y_{2}\right\},\left(y_{1}, y_{2}\right): y_{1} \in \mathbb{T}_{k}^{(2)}(E), y_{2} \in \mathbb{T}_{n-k}^{(2)}(E), k=1, \ldots, n-1\right\}
$$

Set

$$
\mathbb{T}(E)=\cup_{n=1}^{\infty} \mathbb{T}_{n}(E), \quad \mathbb{T}^{(2)}(E)=\cup_{n=1}^{\infty} \mathbb{T}_{n}^{(2)}(E)
$$

When $E=\{1\}$ we write $\mathbb{T}, \mathbb{T}^{(2)}$ instead of $\mathbb{T}(E), \mathbb{T}^{(2)}(E)$.

For $y \in E$ define $\pi(y)=y$ and for $y_{1}, y_{2} \in \mathbb{T}^{(2)}(E)$ define recursively

$$
\pi\left(\left\{y_{1}, y_{2}\right\}\right)=\left\{\pi\left(y_{1}\right), \pi\left(y_{2}\right)\right\}, \quad \pi\left(y_{1}, y_{2}\right)=\pi\left(y_{1}\right)
$$

Then $\pi: \mathbb{T}^{(2)}(E) \rightarrow \mathbb{T}(E)$ is a projection.

Now we define the neighbourhood of a tree in $I^{(2)}$. For $i \in I^{(2)}$, set

$N_{0}(i)=\{i\}, \quad N_{1}^{0}(i)=\{(i, j): j \in I\}, \quad N_{n}^{0}(i)=\left\{(j, k): j \in N_{n-1}^{0}(i), k \in I\right\}$. 
For $i=1, \ldots, N$ set $N_{n}(i)=N_{n}^{0}(i)$ and for $i=\left\{i_{1}, i_{2}\right\}$ set

$$
N_{n}(i)=\left\{j \in N_{n_{0}}^{0}\left(\left\{j_{1}, j_{2}\right\}\right): j_{1} \in N_{n_{1}}\left(i_{1}\right), j_{2} \in N_{n_{2}}\left(i_{2}\right), n_{0}+n_{1}+n_{2}=n\right\}
$$

Make the analogous definition for $i=\left(i_{1}, i_{2}\right)$. Set $N(i)=\cup_{n=1}^{\infty} N_{n}(i)$.

Define $\mathbb{S} \subseteq \mathbb{T}^{(2)}$, the set of surviving trees, by

(i) $1 \in \mathbb{S}$,

(ii) $\left\{\tau_{1}, \tau_{2}\right\} \in \mathbb{S}$ if and only if $\tau_{1} \in \mathbb{S}$ and $\tau_{2} \in \mathbb{S}$,

(iii) $\left(\tau_{1}, \tau_{2}\right) \in \mathbb{S}$ if and only if $\tau_{1} \in \mathbb{S}$ and $\tau_{2} \notin \mathbb{S}$.

We now define some spaces of path-trees. Fix $t>0$ and set $A_{1}[0, t]=C\left([0, t], \mathbb{R}^{d}\right) \times$ $(0, \infty)$. For $\xi=(\omega, y) \in A_{1}[0, t]$

set $\Delta(\xi)=[0, t]$ and define $(x, y)(\xi): \Delta(\xi) \rightarrow \mathbb{R}^{d} \times(0, \infty)$ by $x_{s}(\xi)=\omega_{s}, y_{s}(\xi)=y$. For $\tau=\left\{\tau_{1}, \tau_{2}\right\} \in \mathbb{T}$ define recursively

$$
A_{\tau}[0, t]=\left\{\left(s,\left\{\xi_{1}, \xi_{2}\right\}, \omega\right): s \in(0, t], \xi_{1} \in A_{\tau_{1}}[0, s], \xi_{2} \in A_{\tau_{2}}[0, s], \omega \in C\left([0, t], \mathbb{R}^{d}\right)\right\} .
$$

For $\left(s,\left\{\xi_{1}, \xi_{2}\right\}, \omega\right) \in A_{\tau}[0, t]$, we take $\Delta(\xi)$ to be the disjoint union of $\Delta\left(\xi_{1}\right), \Delta\left(\xi_{2}\right)$ and $[s, t]$. Set $x_{r}(\xi)=x_{r}\left(\xi_{i}\right)$ and $y_{r}(\xi)=y_{r}\left(\xi_{i}\right)$ for $r \in \Delta\left(\xi_{i}\right), i=1,2$. Extend the map $(x, y)(\xi)$ to $\Delta(\xi)$ by setting $x_{r}(\xi)=\omega_{r}, y_{r}(\xi)=y_{s}\left(\xi_{1}\right)+y_{s}\left(\xi_{2}\right)$ for $r \in[s, t]$. Set $A[0, t]=\cup_{\tau \in \mathbb{T}} A_{\tau}[0, t]$. For $\xi \in A[0, t]$ set

$$
A(\xi)=\cup_{s \in \Delta(\xi)}\{s\} \times A[0, s] .
$$

We leave to the reader the analogous definitions for $A^{(2)}[0, t]$.

\section{REFERENCES}

[1] H. Amann, Coagulation-fragmentation processes, Arch. Ration. Mech. Anal., 151(4):339-366, 2000.

[2] O.E. Lanford, III. Time evolution of large classical systems, In dynamical systems, theory and applications (Recontres, Battelle Res. Inst., Seattle, Wash., 1974), Lecture Notes in Phys., 38:1-111. Springer, Berlin, 1975.

[3] R. Lang and X.X. Nguyen, Smoluchowski's theory of coagulation in colloids holds rigorously in the Boltzmann-Grad-limit, Z. Wahrsch. Verw. Gebiete, 54(3):227-280, 1980.

[4] P. Laurençot and S. Mischler, The continuous coagulation-fragmentation equations with diffusion, Arch. Ration. Mech. Anal., 162(1):45-99, 2002.

[5] P. Laurençot and S. Mischler, Global existence for the discrete diffusive coagulationfragmentation equations in $L^{1}$, Rev. Mat. Iberoamericana, 18(3):731-745, 2002.

[6] J.F. Le Gall, Fluctuation results for the Wiener sausage, Ann. Probab., 16(3):991-1018, 1988.

[7] J.R. Norris, Cluster coagulation, Comm. Math. Phys., 209(2):407-435, 2000.

[8] J.R. Norris, Smoluchowski's coagulation equation: uniqueness, nonuniqueness and a hydrodynamic limit for the stochastic coalescent, Ann. Appl. Probab., 9(1):78-109, 1999.

[9] A.S. Sznitman, Propagation of chaos for a system of annihilating Brownian spheres, Comm. Pure Appl. Math., 40(6):663-690, 1987.

[10] A.S. Sznitman, Topics in Propagation of Chaos, in 'Ecole d'Été de Probabilités de Saint-Flour XIX-1989, Lecture Notes in Math., 1464:165-251, Springer, Berlin, 1991.

[11] M.V. Smoluchowski, Drei vorträge über diffusion, brownsche bewegung und koagulation von kolloidteilchen, Physik. Z., 17:557-585, 1916. 